\title{
PJ34, an inhibitor of PARP-1, suppresses cell growth and enhances the suppressive effects of cisplatin in liver cancer cells
}

\author{
SHENG-HUI HUANG ${ }^{1 *}$, MIN XIONG $^{2 *}$, XIAO-PING CHEN ${ }^{1}$, \\ ZHEN-YU XIAO $^{1}$, YIN-FENG ZHAO ${ }^{1}$ and ZHI-YONG HUANG ${ }^{1}$ \\ ${ }^{1}$ Hepatic Surgery Center, Tongji Hospital, ${ }^{2}$ Department of Urology, Union Hospital, Tongji \\ Medical College, Huazhong University of Science and Technology, Wuhan 430030, P.R. China
}

Received April 24, 2008; Accepted May 30, 2008

DOI: $10.3892 /$ or_00000043

\begin{abstract}
It has been suggested that poly(ADP-ribose) polymerase-1 (PARP-l) plays an important role in DNA repair, cell death and proliferation, as well as in the stabilization of the genome. Pharmacological inhibition or genetic ablation of PARP-1 had a beneficial outcome in cancer chemotherapy since the cancer cells lacked PARP-1 and were sensitive to chemotherapeutic DNA damage. As a novel potent specific inhibitor of PARP-1, PJ34 has been reported to enhance chemotherapeutic effects in certain types of tumors. In a previous study, we found that PARP-1 expression was significantly increased in human hepatocellular carcinoma (HCC) compared to its surrounding liver tissue. This study investigated whether or not the inhibition of PARP-1 activity by PJ34 produces suppressive effects on human liver cancer cells and sensitizes the tumor cells to chemotherapeutic agents. We conclude that PJ34 significantly suppresses HepG2 cell growth in a dose-dependent manner, and inhibits HepG2 cellderived tumor growth in nude mice. The suppressive effects of PJ34 are associated with increased cell apoptosis. Furthermore, PJ34 enhances suppressive effects of cisplatin in HepG 2 cells. These results suggest that PJ34 may be developed into an effective agent for the treatment of human HCC.
\end{abstract}

\section{Introduction}

Hepatocellular carcinoma (HCC) is a common malignant disease in Asia and Africa (1). HCC is resistant to most

Correspondence to: Professor Zhi-yong Huang, Hepatic Surgical Center, Tongji Hospital, Tongji Medical College, Huazhong University of Science and Technology, 1095 Jie Fang Da Dao, Wuhan 430030, P.R. China

E-mail: zyhuang@medmail.com.cn

${ }^{*}$ Contributed equally

Key words: poly (ADP-ribose) polymerase-1 inhibitor, PJ34, liver cancer, cisplatin chemotherapeutic protocols and radiation therapy and hepatic resection remains the most effective treatment (2). However, most of the patients present in the late stages of HCC on their first visit and do not have the chance for resection. Even for those who undergo liver resections, postoperative recurrence and tumor metastasis inevitably leads to a poor clinical outcome. To develop potential effective treatments for HCC, recent studies have focused on exploring the molecular mechanisms of HCC formation and progression. HCC is closely linked to persistent HBV infection and the intake of $\alpha$-flatoxin B-contaminated food (3), however, the mechanistic clues for the development of this type of cancer are largely unknown. HCC is hypothesized to represent a multi-stage process resulting from the accumulation of genetic changes $(4,5)$. Different genes have been implicated in the pathogenesis of HCC and genes regulating DNA damage response play an important role in maintaining genomic integrity or leading to the pathogenesis of $\mathrm{HCC}(6)$.

PARP-1 is a $116 \mathrm{kDa}$ nuclear enzyme that catalyzes the poly(ADP-ribosyl)ation of target proteins in response to DNA damage and has been proposed to play an important role in DNA repair, cell death and proliferation, as well as in the stabilization of the genome $(7,8)$. Overexpression of PARP-1 has been reported in various human malignancies, such as malignant lymphoma (9), breast carcinoma (10), Ewing's sarcoma (11) and HCC (12-14). Previous studies demonstrated that pharmacological inhibition or genetic ablation of PARP-1 not only provided remarkable protection from tissue injury in various oxidative stress-related disease models but resulted in a clear beneficial outcome in the treatment of cancer (15). In certain types of tumors including lung cancer, colon carcinoma and HeLa cells, PARP-1 inhibition was shown to be an effective means of enhancing tumor sensitivity to radiation and chemotherapy (16-19). Pharmacological inhibition of PARP-1 with 3,4-dihydro-5-[4-(1piperidinyl)butoxyl]-1(2H)-isoquinolinone (DPQ) results in a delay in tumor formation and in a dramatic reduction in tumor size and multiplicity during 7,12-dimethylbenz(a)anthracene plus 12-O-tetradecanoylphorbol-13-acetateinduced skin carcinogenesis (20), which suggests that PARP-1 inhibitors not only increase tumor cell sensitivity to radiation and chemotherapy, they also suppress tumor growth 
in certain types of tumors. However, whether or not PARP-1 inhibition is able to suppress tumor cell growth and whether it improves chemotherapeutic effects in human liver cancer remains unknown.

PJ34 [N-(6-oxo-5, 6-dihydrophenanthridin-2-yl)-N, Ndimethylacetamide $\mathrm{HCl}$ ] is a newly-generated specific inhibitor of PARP-1. PJ34 has demonstrated wide-spread therapeutic activity in suppressing the inflammatory response in colitis, autoimmune diabetes and uveitis $(21,22)$ and in a number of models of ischemia (21). Additionally, PJ-34 was found to reduce VEGF-induced proliferation, migration and tube formation of human umbilical vein endothelial cells in vitro in a dose-dependent manner, which suggested that the treatment with PJ34 may exert additional benefits in various cancers by decreasing angiogenesis (23). In this study, the suppressive effects of PJ34 alone and PJ34 in combination with a chemotherapeutic agent in human liver cancer cell lines have been investigated in vivo and in vitro and the role of the PARP-1 inhibitor, PJ34, in human liver cancer therapy has been evaluated.

\section{Materials and methods}

Cell lines and culture. Human liver cancer cell lines, HepG2 and SMMC7721, and the normal human hepatocyte line L02 were maintained by the Hepatic Surgery Center, Tongji Hospital, Tongji Medical College. The cell lines were cultured with Dulbecco's modified Eagle's medium (DMEM, Sigma), containing $10 \%$ fetal bovine serum supplemented with penicillin $(100 \mathrm{U} / \mathrm{ml})$ and streptomycin $(100 \mu \mathrm{g} / \mathrm{ml})$. The cell lines were maintained at $37^{\circ} \mathrm{C}$ in a humidified incubator with $95 \%$ air and $5 \% \mathrm{CO}_{2}$.

Cell proliferation assay. To establish cell growth curves, $5 \times 10^{4}$ cells were seeded in 18 plates with a $6-\mathrm{cm}$ diameter. The plates were divided into two groups, each of which had nine plates. One group was cultured with DMEM only, while the other group was cultured with DMEM plus PJ34 at different concentrations. The medium was changed every 2-3 days. Cell numbers were counted on days 3, 6 and 9 after seeding. The optimal concentration of PJ34 was determined by pre-culturing the HepG2 cells with different concentrations of PJ34 at 0.5, 1.0 and $2.0 \mathrm{mg} / 1$, respectively. The optimal concentration was chosen as $1.0 \mathrm{mg} / \mathrm{l}$ as it was the highest concentration at which the normal liver cell line L02 cells survived well and no drug-related cell death was observed in the culture. The assay was repeated three times.

Cell apoptosis analysis. The same number of HepG2 cells were seeded in 10 plates with a $6-\mathrm{cm}$ diameter. Five plates were cultured with a regular medium containing $1.0 \mathrm{mg} / \mathrm{l}$ of PJ34, while the other five plates had regular medium only. Cells were harvested at $0,12,24,48$ and $72 \mathrm{~h}$ after incubation. Cells were suspended twice with ice cold PBS. The cell densities were adjusted to $1-2 \times 10^{6}$ cells $/ \mathrm{ml}$ in each tube. The chilled $80 \%$ methanol was added to each tube and stored at $-20^{\circ} \mathrm{C}$ overnight. The tubes were centrifuged and the methanol was removed. PI was added ( $1 \mathrm{ml})(5 \mathrm{mg}$ propidium iodine, $0.1 \mathrm{ml}$ Triton X-100, $3.7 \mathrm{mg}$ EDTA in $100 \mathrm{ml}$ PBS) and $10.1 \mu \mathrm{l}$ of RNase solution (100 U/ml ) and mixed gently and incubated in a dark at room temperature for $30 \mathrm{~min}$. The samples were subjected to a flow cytometer (Becton Dickinson Immunocytometry Systems, USA) for analysis within 1 hour.

Animals and tumor growth. Fifteen male balb/c nude mice were provided by the Experimental Animal Center of Tongji Medical College. Mice were 8 weeks old and weighed between 18 and $20 \mathrm{~g}$. Exponentially growing cells were harvested and $2 \times 10^{6}$ HepG 2 cells were injected into each of the bilateral flanks of nude mice. The HepG2-derived tumors were formed around day 14 after injection. Tumor-bearing mice were randomly divided into two groups, the PJ34 group with 8 mice and the control group with 7 mice. The PJ34 group had 15 tumors, while the control group had 12 tumors. $\mathrm{PJ} 34$ at $3 \mathrm{mg} / \mathrm{kg}$ in $0.1 \mathrm{ml}$ was injected intraperitoneally into the nude mice in the PJ34 group every other day for 21 days, while the same volume of saline was injected into the mice in the control group at the same time interval. The tumors were measured using a vernier caliper every 3-4 days. Tumor sizes were calculated on day 21 after using the following formula: average diameter $=$ (longitudinal diameter + transverse diameter) $/ 2$.

Chemopotentiation assay. HepG2 cells $\left(5 \times 10^{4}\right)$ were seeded in 27 plates with a 6 -cm diameter. The plates were divided into three groups, where each group had nine plates. One group was cultured with DMEM plus cisplatin at the concentration of $0.25 \mathrm{mg} / \mathrm{l}$ and the second group was cultured with DMEM plus PJ34 at $1.0 \mathrm{mg} / \mathrm{l}$, while the third group had DMEM plus cisplatin at $0.25 \mathrm{mg} / \mathrm{l}$ and PJ34 at $1.0 \mathrm{mg} / \mathrm{l}$. The medium was changed every 2-3 days. Cell numbers were counted on days 3,6 and 9 after seeding. The assay was repeated three times. The average cell numbers were determined for each counting day in each group.

Statistical methods. Data are presented as the mean \pm SD and were analyzed by the t-test, with significance (P) set at $<0.01$.

\section{Results}

PJ34 inhibits the growth of liver cancer cell lines, but not the normal liver cell line in vitro. When HepG2 cells were cultured with PJ34 at concentrations of $0.5,1.0$ and $2.0 \mathrm{mg} / 1$, HepG2 cell growth was significantly suppressed compared to those cells cultured without PJ34. As the concentration of PJ34 was increased, its suppressive effects were gradually enhanced (Fig. 1A). There was a significant difference in the cell numbers between the group without PJ34 and the group with the different concentrations of PJ34 on days 6 and 9 of culture $(\mathrm{P}<0.01)$. To further study the suppressive effects of PJ34 on HCC cells, SMMC7721 cells were cultured with PJ34 at $1.0 \mathrm{mg} / \mathrm{l}$ for 9 days, where there was a significant difference in the cell numbers between the group with PJ34 and the group without PJ34 on days 6 and 9 of culture (Fig. 1B, $\mathrm{P}<0.01)$. However, when the human normal liver cell line, L02, was cultured with PJ34 at $1.0 \mathrm{mg} / 1$, no suppressive effect was observed on days 3, 6 and 9 of culture (Fig. 1C, P>0.05).

The suppressive effects of PJ34 is associated with increased cell apoptosis. To explore the potential mechanism by which 


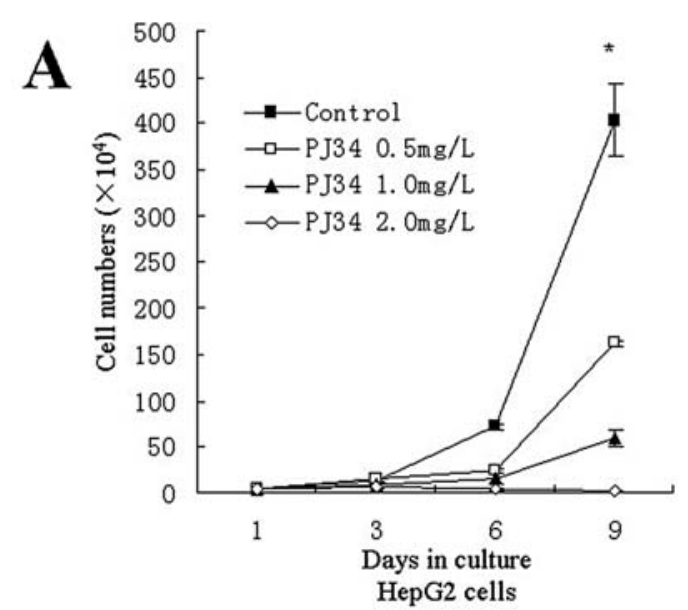

C

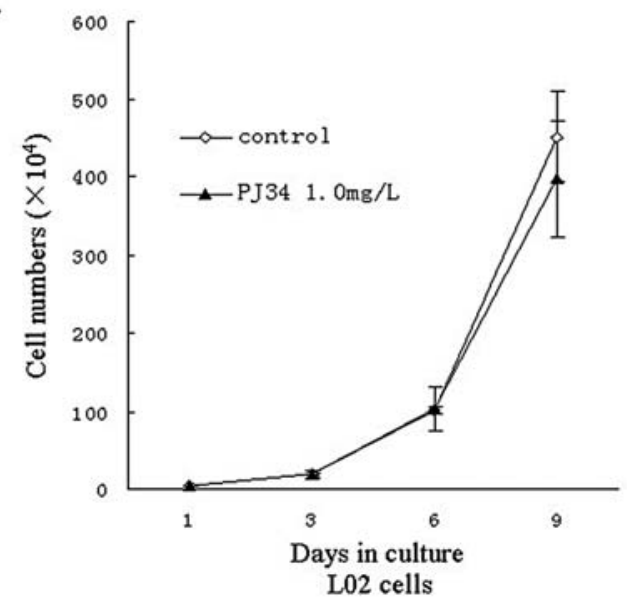

Figure 1. PJ34 inhibits HepG2 and SMMC7721 cell growth in vitro. PJ34 at $0.5,1.0$ and $2.0 \mathrm{mg} / 1$ significantly inhibits HepG2 cell proliferation on days 6 and 9 of culture (A) and PJ34 at $1.0 \mathrm{mg} / 1$ suppresses SMMC7721 cell proliferation on days 6 and 9 of culture (B). However, PJ34 at $1.0 \mathrm{mg} / \mathrm{l}$ does not suppress L02 cell proliferation $(\mathrm{C}) .{ }^{*}$ Statistically different $(\mathrm{P}<0.01)$.

PJ34 suppresses HepG2 cell growth, cell apoptosis indices of the PJ34-treated HepG2 cells and the untreated HepG2 cells were investigated. Flow cytometry analysis showed that the apoptosis indices of HepG2 cells at 0, 12, 24 and $48 \mathrm{~h}$ of culture in the PJ34-treated HepG2 cells were comparable to those untreated HepG2 cells at the same time points of culture, however, the apoptosis index of the untreated-HepG2 cells at $72 \mathrm{~h}$ of culture was significantly higher than that in the untreated HepG2 cells (Fig. 2A). There was a significant difference in the apoptosis index at $72 \mathrm{~h}$ of culture between the PJ34-treated HepG2 cells and the untreated HepG2 cells $(\mathrm{P}<0.01)$ (Fig. 2B).

PJ34 inhibits HepG2 cell-derived tumor growth in nude mice. To confirm the suppressive effects of PJ34 on HepG2 in vivo, $2 \times 10^{6}$ of the HepG2 cells were injected into each of the bilateral flanks of the nude mice. Tumors formed after two weeks, and tumor-bearing mice were randomly divided into two groups, the PJ34 group and the control group. PJ34 at 3 mg per $\mathrm{kg}$ of mouse weight was injected intraperitoneally into each nude mouse of the PJ34 group every other day for 21 days, while the same volume of only saline was injected into the mice of the control group. The tumor sizes of the PJ34 group were significantly smaller than those of the control

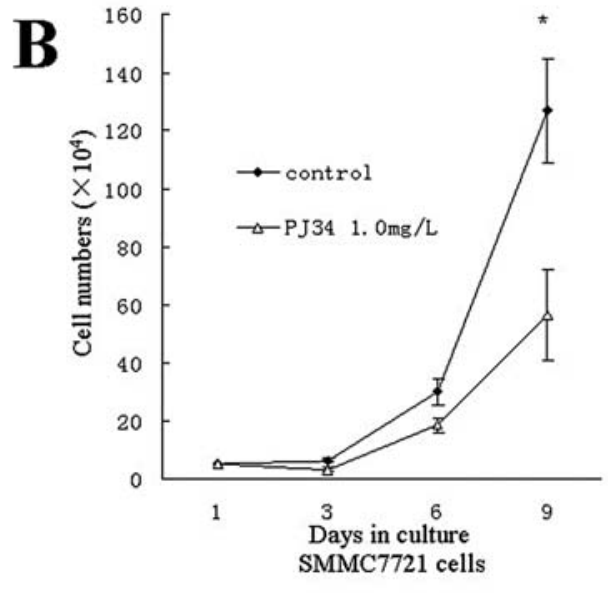

group (Fig. 3A). There was a significant difference in tumor size between the two groups $(\mathrm{P}<0.05)$ (Fig. 3B).

PJ34 enhances the suppressive effects of cisplatin in HepG2 cells. Either cisplatin at $0.25 \mathrm{mg} / \mathrm{l}$ or PJ34 at $1 \mathrm{mg} / \mathrm{l}$ alone inhibited HepG2 cell growth. There was a significant difference in the cell numbers between the cells with cisplatin or PJ34 and the cells with a regular medium on days 6 and 9 of culture. However, when cisplatin (at $0.25 \mathrm{mg} / \mathrm{l}$ ) was combined with PJ34 (at $1 \mathrm{mg} / \mathrm{l}$ ), the suppressive effects of cisplatin were significantly enhanced, there was a significant difference in the cell numbers between the cells cultured with cisplatin or PJ34 and the cells cultured with the combination of PJ34 and cisplatin on days 3, 6 and 9 of culture (Fig. 4A). The cell growth suppression rates in the HepG2 cells cultured with the combination of PJ34 and cisplatin were significantly higher than those in the HepG2 cells with cisplatin or PJ34 only on days 6 and 9 of culture (Fig. 4B), and there was a significant difference in the suppression rates between the HepG2 cells with cisplatin or PJ34 only and those with both PJ34 and cisplatin $(\mathrm{P}<0.01)$.

\section{Discussion}

It has been suggested that HCC results from the accumulation of genetic changes (4) and genes regulating DNA damage response such as PARP-1 and the Ku80 gene plays an important role in maintaining genomic integrity or leading to the pathogenesis of HCC. Recent studies have indicated that haplo-insufficiency of Ku80 in PARP-1\% mice promotes the development of hepatocellular adenoma and HCC (6), which suggests that PARP-1 deletion plays an important role in the pathogenesis of HCC. Theoretically, after a cell suffers from DNA damage, it is imperative for a cell to accurately repair its DNA after damage and to return to a normal cellular phenotype. If cell DNA damage can not be repaired completely and with high fidelity, some severely damaged cells can survive as neoplastic transformants. In certain human cancers, however, a high frequency of DNA lesions and an overactived PARP-1 pathway were observed. In this regard, PARP-1 was implicated in playing a protective role against the accumulated DNA damage of tumor cells, PARP-1 activation may be vital for tumor cells surviving from accumulated DNA damage. Thus, the inhibition of 

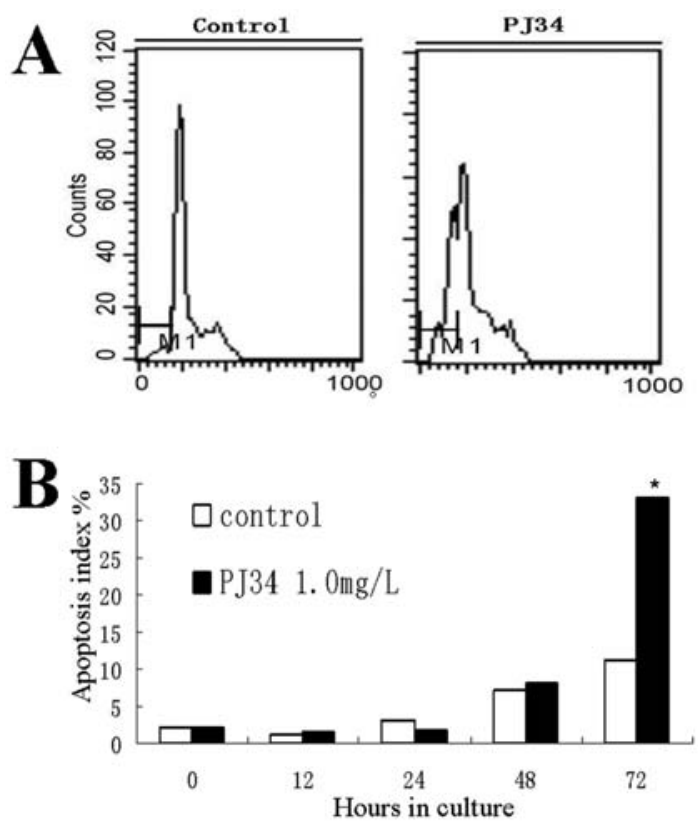

Figure 2. The flow cytometry analysis shows that the apoptosis area (denoted by M1) of the HepG2 in the PJ34 group is more significant than that in the control group at $72 \mathrm{~h}$ of culture (A). At $72 \mathrm{~h}$ of culture, HepG2 cells cultured with $1.0 \mathrm{mg} / \mathrm{l}$ of PJ34 exhibit a higher apoptosis index than the HepG2 cells cultured without PJ34 (B). *Statistically significant difference ( $<<0.01)$.

PARP-1 activation may result in tumor cell death or enhanced sensitivity of tumor cells to further DNA damage caused by radiation and chemotherapy. Previous studies have indicated that pharmacological inhibition of PARP-1 inhibits tumor development in skin cancer (19). PARP-1 inhibition also shows promise as an effective means of enhancing tumor
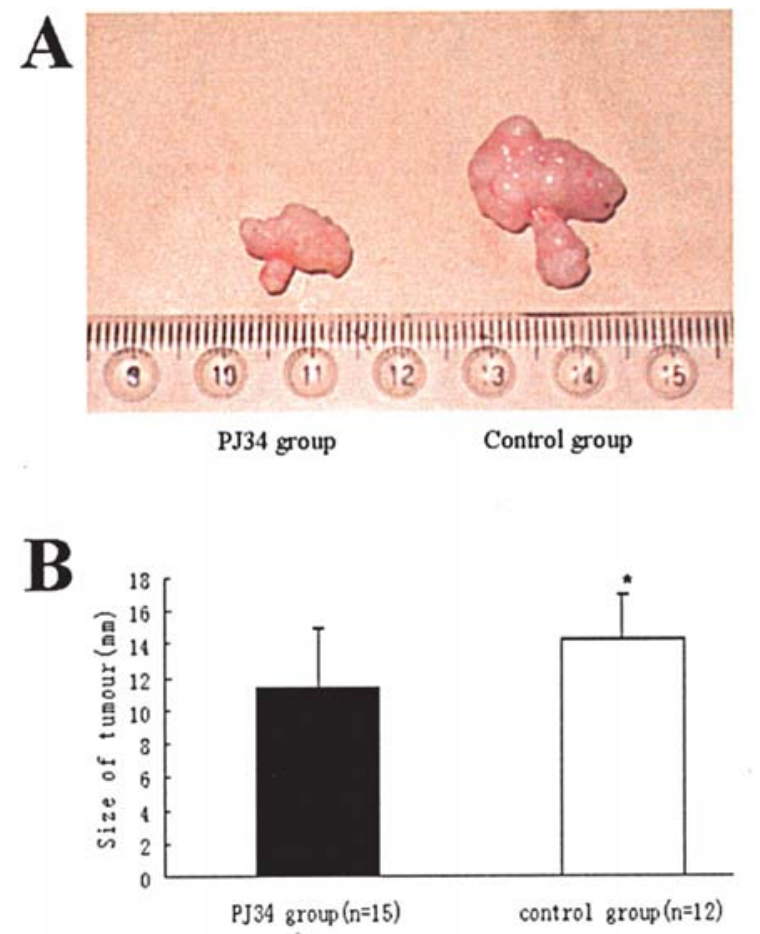

Figure 3. PJ34 significantly inhibits HepG2 cell-derived tumor growth in nude mice. The tumors of the PJ34 group were significantly smaller than those in the control group (A). "Statistical difference in tumor size between the two groups (B).

sensitivity to radiation and chemotherapy for lung cancer, colon carcinoma, HeLa cells and intracranial melanoma, glioma and lymphoma (15-18,24). In HCC, PARP-1 expression and activity were significantly increased compared to their
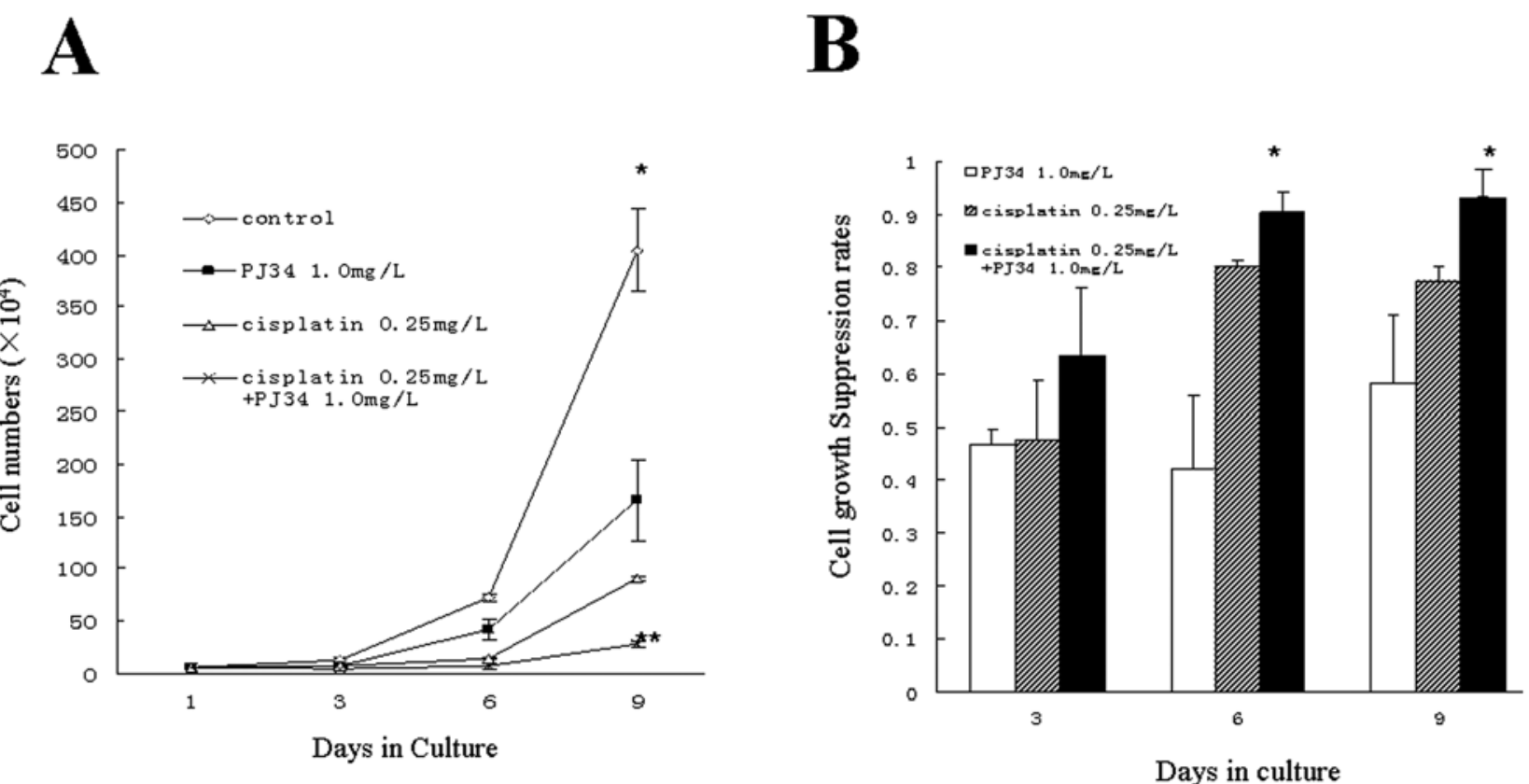

Figure 4. PJ34 enhances the suppressive effect of cisplatin in HepG2 cell growth. Either cisplatin at $0.25 \mathrm{mg} / \mathrm{l}$ or PJ34 at $1 \mathrm{mg} / 1$ alone inhibited HepG2 cell growth on days 6 and 9 of culture. There was a statistical difference in the cell numbers between the control group and the group with cisplatin or PJ34 on day 9 of the culture $\left({ }^{* *} \mathrm{p}<0.01\right)$ and in association with PJ34, the suppressive effects of cisplatin were significantly enhanced on days 6 and 9 of culture. There was a statistical difference in the cell numbers between the group with cisplatin plus PJ34 and the group with cisplatin or PJ34 on day 9 of culture $\left({ }^{* *} \mathrm{P}<0.01\right)(\mathrm{A})$. HepG2 cell growth suppression rates of the group with PJ34 plus cisplatin are significantly higher than that of the group with cisplatin or PJ34 on days 6 and 9 of culture (B). There was a significant difference in the growth inhibition rate between the two groups on day 9 of culture $\left({ }^{*} \mathrm{P}<0.01\right)$. 
corresponding adjacent non-tumor liver tissue (12-14), which suggested that PARP-1 inhibition may have a therapeutic significance in HCC.

Our study suggested that PJ34 alone was able to inhibit the growth of the liver cancer cell line, HepG2 and SMMC7721. In HepG2 cells, the suppressive effects of PJ34 were observed in a dose-dependent manner. In order to exclude the effects of cellular toxicity produced by PJ34 itself, the normal liver cell L02 cell line was used as a control and no suppressive effects on the growth of normal liver L02 cells was found. Further studies indicated that growth suppression mediated by PJ34 was associated with increased apoptotic rates in HepG2 cells. In addition, PJ34 was able to inhibit hepG2derived tumor growth in nude mice. These observations suggest that PJ34 alone is able to suppress liver cancer cell growth in vitro and in vivo. However, the ability of DNA repair inhibitors in preventing cancer development is still difficult to interpret. Inhibitors may act on multiple aspects of DNA repair as well as the cellular signaling pathways activated in response to the initial damage. The possible mechanisms that have been suggested include the selective killing of homologous recombination-deficient tumor cells, the down-regulation of tumor-related gene expression and a decrease in the apoptotic threshold in the co-treatment with chemo and radiotherapy (15). Some studies have indicated that PARP-1 inhibition prevented tumor promotion through their ability to cooperate with the activation of AP-1, NF- $\kappa \mathrm{B}$, and HIF-1 $\alpha$ (20). Our data demonstrated that the growth suppression of HepG2 cells mediated by the PARP-1 inhibitor, PJ34, was associated with increased cell apoptosis.

Cisplatin is one of the most widely used antitumor drugs and demonstrates the phenomenon of drug resistance (intrinsic or acquired) against a wide variety of tumors including liver cancer. By inhibiting Poly(ADP-ribose) polymerase-1, the chemosensitivity of cisplatin to tumor cells can be increased so that cisplatin resistance can be circumvented. For example, PARP-1 inhibitor 3-aminobenzamide (3-AB) increases the cytotoxic activity of the platinum compounds cisplatin, trans-[PtCl(2)(4-picoline)(piperazine)] and transplatin against $\mathrm{CH} 1$ cisR cisplatin-resistant ovarian tumor cells (25). Our study indicated that PJ34 significantly enhanced the suppressive effects of cisplatin in HepG2 cells.

In summary, our studies demonstrated that the PARP-1 inhibitor, PJ34, was able to suppress cell growth of liver cancer cell lines in vitro and HepG2-derived tumor development in vivo. In addition, PJ34 enhanced the suppressive effects of cisplatin in HepG2 cells. This finding exerts a profound impact in the search for a more effective treatment for human liver cancer. Future studies will be focused on clinical trails of the PARP-1 inhibitor, PJ34, for the treatment of HCC and will further evaluate its clinical value.

\section{Acknowledgements}

This study was supported by funds from the National Natural Science Foundation of China (No. 30772126) and the New Century Excellent Talent Foundation of China Ministry of Education (No. NCET-04-0701) to Dr Zhi-Yong Huang.

\section{References}

1. Chen XP and Huang ZY: Surgical treatment of hepatocellular carcinoma in China: surgical techniques, indications, and outcomes. Langenbecks Arch Surg 390: 259-265, 2005.

2. Chen XP, Qiu FZ, Wu ZD, Zhang ZW, Huang ZY and Chen YF: Long-term outcome of resection of large hepatocellular carcinoma. Br J Surg 93: 600-606, 2006.

3. Chen CJ, Yu MW and Ye L: Epidemiological characteristics and risk factors of hepatocellular carcinoma. J Gastroenterol Hepatol 12: S294-S308, 1997.

4. Suriawinata A and Xu R: An update on the molecular genetics of hepatocellular carcinoma. Semin Liver Dis 24: 77-88, 2004.

5. Röcken C and Carl-McGrath S: Pathology and pathogenesis of hepatocellular carcinoma. Dig Dis 19: 269-278, 2001.

6. Tong WM, Cortes U, Hande MP, et al: Synergistic role of Ku80 and poly(ADP-ribose) polymerase in suppressing chromosomal aberrations and liver cancer formation. Cancer Res 62: 6990-6995, 2002.

7. Tong WM, Ohgaki H, Huang H, Granier C, Kleihues P and Wang ZQ: Null mutation of DNA strand break-binding molecule poly-(ADP-ribose) polymerase causes medulloblastomas in p53(-/-)mice. Am J Pathol 162: 343-350, 2003.

8. Tong WM, Hande MP, Lansdorp PM and Wang ZQ: DNA strand break-sensing molecule poly(ADP-ribose) polymerase cooperates with p53 in telomere function, chromosome stability, and tumor suppression. Mol Cell Biol 21: 4046-4053, 2001.

9. Tomoda T, Kurashige T, Moriki T, Yamamoto H, Fujimoto S and Taniguchi T: Enhanced expression of poly(ADP-ribose) synthetase gene in malignant lymphoma. Am J Hematol 37: 223-227, 1991.

10. Hu JJ, Roush GC, Dubin N, Berwick M, Roses DF and Harris MN: Poly(ADP-ribose) polymerase in human breast cancer: a casecontrol analysis. Pharmacogenetics 7: 309-316, 1997

11. Prasad SC, Thraves PJ, Bhatia KG, Smulson ME and Dritschilo A: Enhanced poly(adenosine diphosphate ribose) polymerase activity and gene expression in Ewing's sarcoma cells. Cancer Res 50: $38-43,1990$

12. Nomura F, Yaguchi M, Togawa A, et al: Enhancement of polyadenosine diphosphate-ribosylation in human hepatocellular carcinoma. J Gastroenterol Hepatol 15: 529-535, 2000.

13. Shiobara M, Miyazaki M, Ito H, et al: Enhanced polyadenosine diphosphate-ribosylation in cirrhotic liver and carcinoma tissues in patients with hepatocellular carcinoma. J Gastroenterol Hepatol 16: 338-344, 2001.

14. Wang ZQ, Huang ZY, Chen XP and Zhang ZF: The expression of Poly(ADP-ribose) polymerase and its biological significances in human hepatocellular carcinoma. World Chin J Dig 14: 1995-1998, 2006.

15. Aguilar-Quesada R, Muñoz-Gámez JA, Martín-Oliva D, et al: Modulation of transcription by PARP-1: consequences in carcinogenesis and inflammation. Curr Med Chem 14: 1179-1187, 2007.

16. Albert JM, Cao C, Kim KW, et al: Inhibition of poly(ADP-ribose) polymerase enhances cell death and improves tumor growth delay in irradiated lung cancer models. Clin Cancer Res 13: 3033-3042, 2007

17. Donawho CK, Luo Y, Luo Y, et al: ABT-888, an orally active poly(ADP-ribose) polymerase inhibitor that potentiates DNAdamaging agents in preclinical tumor models. Clin Cancer Res 13: 2728-2737, 2007.

18. Tentori L, Leonetti C, Scarsella M, et al: Inhibition of poly (ADP-ribose) polymerase prevents irinotecan-induced intestinal damage and enhances irinotecan/temozolomide efficacy against colon carcinoma. FASEB J 20: 1709-1711, 2006.

19. Noël G, Godon C, Fernet M, Giocanti N, Mégnin-Chanet F and Favaudon V: Radiosensitization by the poly(ADP-ribose) polymerase inhibitor 4-amino-1,8-naphthalimide is specific of the $\mathrm{S}$ phase of the cell cycle and involves arrest of DNA synthesis. Mol Cancer Ther 5: 564-574, 2006.

20. Martin-Oliva D, Aguilar-Quesada R, O'valle F, et al: Inhibition of poly(ADP-ribose) polymerase modulates tumor-related gene expression, including hypoxia-inducible factor- 1 activation, during skin carcinogenesis. Cancer Res 66: 5744-5756, 2006.

21. Black JH, Casey PJ, Albadawi H, Cambria RP and Watkins MT: Poly adenosine diphosphate-ribose polymerase inhibitor PJ34 abolishes systemic proinflammatory responses to thoracic aortic ischemia and reperfusion. J Am Coll Surg 203: 44-53, 2006. 
22. Hamby AM, Suh SW, Kauppinen TM and Swanson RA: Use of a poly(ADP-ribose) polymerase inhibitor to suppress inflammation and neuronal death after cerebral ischemia-reperfusion. Stroke 38: 632-636, 2007.

23. Rajesh M, Mukhopadhyay P, Bátkai S, Godlewski G, Haskó G, Liaudet L and Pacher P: Pharmacological inhibition of poly (ADP-ribose) polymerase inhibits angiogenesis. Biochem Biophys Res Commun 350: 352-357, 2006.

24. Tentori L, Leonetti C, Scarsella M, et al: Systemic administration of GPI 15427, a novel poly(ADPribose) polymerase-1 inhibitor, increases the antitumor activity of temozolomide against intracranial melanoma, glioma, lymphoma. Clin Cancer Res 9: 5370-5377, 2003.
25. Nguewa PA, Fuertes MA, Cepeda V, Alonso C, Quevedo C, Soto $\mathrm{M}$ and Pérez JM: Poly(ADP-ribose) polymerase-1 inhibitor 3-aminobenzamide enhances apoptosis induction by platinum complexes in cisplatin-resistant tumor cells. Med Chem 2: 47-53, 2006 . 